\title{
Eggs alone
}

\section{Human parthenotes: an ethical source of stem cells for therapies?}

\section{Ann A. Kiessling}

ए ggs are enormous cells in a state of arrested expectancy. Before the advent of assisted reproductive technologies, human eggs played out their fate in the privacy of the ovary, fallopian tubes and uterus. Women are born with around one million eggs, but by 55 years of age, all these are gone. The loss appears to be relatively uniform throughout life - each year approximately 20,000 eggs die in the ovary, whereas just 12 mature and ovulate. Once released into the fallopian tube, an egg that encounters sperm is fertilized and begins the rapid cycles of cell division that are characteristic of the normal embryo. It completes its journey to the uterus, where it develops the small group of stem cells with the potential to differentiate into all tissue types, a characteristic known as 'pluripotency'. Eggs not fertilized by sperm were simply presumed to disintegrate.

But eggs can activate on their own without sperm, a process known as 'parthenogenesis', from the Greek for 'virgin'. Although some insect species produce workers by parthenogenesis, mammalian parthenotes fail to develop into offspring. Mouse and rabbit parthenotes appear morphologically indistinguishable from fertilized eggs for the first few days of development, including the formation of the small group of pluripotent stem cells. But these parthenotes are not viable and fail to develop into offspring. Two types of growths - dermoid cysts of the ovary and teratomas - suggest that human eggs can also undergo parthenogenesis. Dermoid cysts arise if the ovarian sack fails to rupture; the retained egg activates cell division on its own and gives rise to a small cluster of cells that undergo limited differentiation. Teratomas are benign growths that are not restricted to the ovary and exhibit a variety of cell types, including skin, muscle, bone and occasionally hair and rudimentary teeth.

Long regarded as curious, unimportant quirks of nature, teratomas and ovarian dermoids provide evidence that human parthenogenesis does occur and that human parthenotes spontaneously give rise to at least a few pluripotent stem cells.

Embryonic stem cells from mice have been used as experimental tools for more than two decades, but widespread recognition of the therapeutic potential of pluripotent human stem cells is more recent. Stem-cell therapy offers hope for diseases that result from a lack of a tissue reservoir of stem cells to replace defective and dying cells, such as heart failure, diabetes, Parkinson's

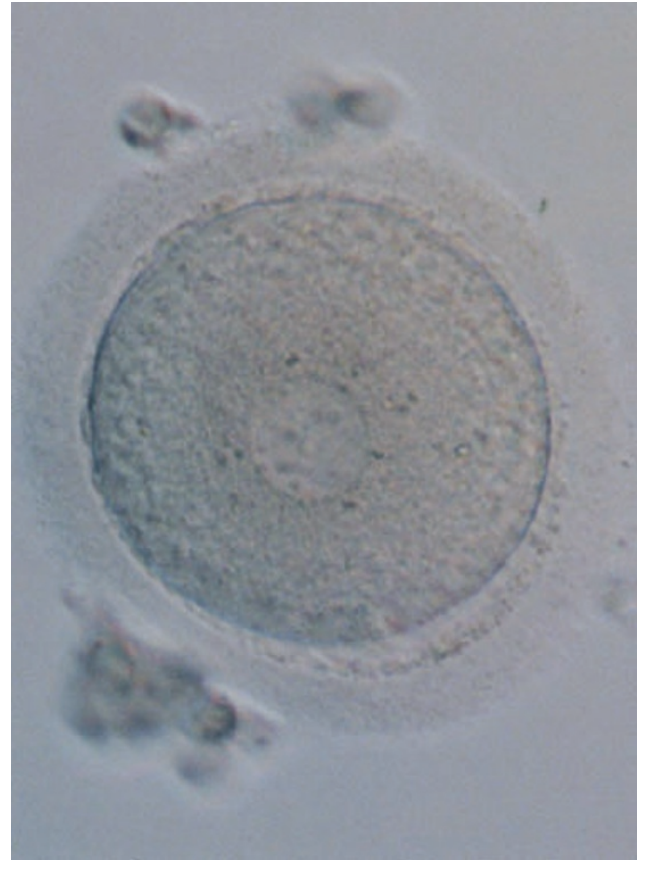

A human parthenote 12 hours after activation.

disease, spinal-cord injury, AIDS and perhaps Alzheimer's disease. But the use of human embryos as a source of stem cells has proved morally and ethically contentious, and led to bans on human embryo research in several countries, including the United States. Could human parthenotes provide an alternative to the use of fertilized eggs?

One caveat to the potential therapeutic value of parthenote stem cells centres on the very reason that mammalian parthenotes do not develop to offspring. Genes inherited from the mother are expressed differently during embryonic development from genes that are inherited from the father. This trait is termed genetic 'imprinting', and it is not known with certainty that stem cells carrying only maternally imprinted genes will behave as robustly as embryonic stem cells. However, it is encouraging that the one line of monkey parthenote stem cells that has been reported (Cyno-1) seems to have the same potential as embryonic stem cells to continually divide and to develop into all cell types.

At present, the only source of human eggs is the human ovary. Is it possible to rescue some of the thousands of doomed eggs for use as parthenote stem cells? Or do these considerations simply replace the moral dilemma of using human embryos for obtaining stem cells with the moral dilemma of collecting eggs from the ovaries of women for therapeutic instead of reproductive purposes? The derivation of stem-cell lines from parthenotes depends on the availability of human eggs and on developing the technology to activate them in the laboratory and promote cell division to stem cells. Proof of the principle was provided by two reports, but no stem-cell lines were derived.

For women with serious diseases, such as Type 1 diabetes or spinal-cord injury, using their eggs to generate stem cells for their own potential therapeutic use requires little justification. The process could be likened to self-donation of bone-marrow stem cells, or blood before a surgical procedure. Because genetic information is exchanged between paired chromosomes during egg maturation, parthenote stem cells may not be genetically identical to the woman, but will undoubtedlybe a closer tissue match than stem cells from human embryos.

Moreover, it is theoretically possible that such parthenote stem-cell lines could also be made available to other tissue-matched individuals, including men or older women. In this way, parthenote stem-cell banks could replace the need for the ethically controversial embryonic stem-cell banks.

A recent elegant experiment has added to the confusion about the 'embryo' status of parthenotes. Genetically engineered mouse egg chromosomes were shown to behave like mouse sperm chromosomes when transferred into an unfertilized mouse egg. The development of the resulting reconstructed egg was titled 'parthenogenesis', although it differed markedly from canonical parthenogenesis, which relies solely on the egg's own chromosomes.

Manipulating human eggs to give rise to stem cells for therapies instead of embryos for reproduction requires society to expand its understanding of the capabilities of eggs. New terms to accurately describe the new tasks will make it easier for policy makers to understand and establish appropriate guidelines for human egg research, including the promise of parthenotes.

Ann A. Kiessling is at the Harvard Institutes of Medicine, 4 Blackfan Circle, Boston,

Massachusetts 02115, USA.

\section{FURTHER READING}

Kiessling, A. A. \& Anderson S. C. Human Embryonic Stem Cells (Jones and Bartlett, Sudbury, 2003).

Kiessling, A. A. Connecticut Law Rev., 36, 1051-1092 (2004).

Cibelli, J. B. et al. Science 295, 819 (2002).

Kono, T. et al. Nature 428, 860-864 (2004). 